\title{
Bestimmung von Oxocarbonsäuren im Urin
}

\author{
Von H.-Ch. Curtius, A. Niederwieser und U. Redweik \\ Aus der Medizinisch-Chemischen Abteilung der Universitäts-Kinderklinik Züiricb \\ (Direktor: Prof. Dr. A. Prader)
}

(Eingegangen am 7. Dezcmber 1971/26. April 1972)

Es wird eine Methode zur quantitativen Bestimmung von Oxosäuren im Urin beschrieben. Dic Oxosäuren wcrden zunächst bei einem $\mathrm{pH}$ von 2,5 bis 3 in ihre 2,4-Dinitrophenylhydrazone umgewandelt, diese anschließend mit $\mathrm{H}_{2} / \mathrm{PtO}_{2}$ in $\mathrm{A}$ thanol zu den entsprechenden Aminosäuren reduziert und mit Hilfe des automatischen Aminosäuren-Analysators nach SterN und Moore bestimmt.

Die Ausbeute von $11 \mathrm{dem}$ Urin zugemischten Oxosäuren wurde bestimmt. Die Brauchbarkeit der Methode wird am Beispiel eines Normalurins, dem Urin eines Patienten mit Phenylketonurie und eines Patienten mit Leucinose (Verzweigtketten-Oxoacidurie) illustriert.

\section{Determination of urinary keto acids}

A method for the quantitative determination of urinary keto acids is described. The keto acids are converted into their 2,4-dinitrophenylhydrazones at $\mathrm{pH} 2.5$ to 3 , reduced to their corresponding amino acids by means of $\mathrm{H}_{2} / \mathrm{PtO}_{2}$ in ethanol and quantitatively determined according to STEIN and MOORE. The recovery of 11 keto acids was measured.

The results obtained with this method are presented for a normal subject, a patient with phenylketonuria and a patient with leucinosis (branched-chain oxoaciduria).

Die 2-Oxosäuren spielen eine bedeutende Rolle im Aminosäure- und Fettsäure-Stoffwechsel. Die Analyse der Oxosäuren im Urin ist daher von großem biochemischen und medizinischen Interesse; bei gewissen angeborenen Stoffwechselkrankheiten wie Phenylketonurie, Leucinose und Histidinämie ist die Ausscheidung einiger dieser Säuren massiv erhöht. Ihre spezifische und quantitative Bestimmung ist für die Diagnose dieser Krankheiten von großer Bedeutung. Die spezifische Analyse der Oxosäuren stellt ein komplexes Problem dar. Im Urin sind eine Vielzahl von organischen Säuren nachweisbar, zudem sind die Oxosäuren teilweise wasserlöslich und instabil. Die bis heute üblichen Methoden beruhen entweder auf der papier- oder dünnschichtchromatographischen Trennung der 2,4-Dinitrophenylhydrazone (1-4), der Gaschromatographie der Methoximtrimethylsilyläther (5) oder der Chinoxalone (6). KeSNER und Mitarbeiter (7) beschrieben die Trennung der Krebszyklus-Säuren auf Silicagel.

Die meist verwendeten papier- und dünnschichtchromatographischen Methoden der 2,4-Dinitrophenylhydrazone sind allerdings nur sehr beschränkt brauchbar. Abgesehen von der Bildung von jeweils zwei Stereoisomeren sind die Dinitrophenylhydrazin-Derivate der Oxosäuren äußerst labil und können nur bei $4^{\circ}$ unzersetzt chromatographiert werden. Eine saubere Trennung gelingt nur bei Testsubstanzen (8). Die Gaschromatographie dieser Verbindungen ist ebenfalls nur beschränkt anwendbar, da die Extraktion der teilweise sehr stark wasserlöslichen Substanzen problematisch ist. Die Kolonnenchromatographie an Kieselgel, wie sie von KESNER beschrieben wurde, ergibt in biologischem Material keine vollständige Auftrennung.

Towers, THOMpson und Steward haben schon 1954 eine Methode zur katalytischen Hydrierung der 2,4Dinitrophenylhydrazone zu den entsprechenden Aminosäuren beschrieben (9). Diese Methode wurde 1956 von MeIster und AbendsChein nochmals verbessert (10). Die Hydrierung wurde in neutralem Milieu in wäßriger Lösung durchgeführt und die Aminosäuren mittels Papierchromatographie bestimmt. Die an sich sehr elegante Technik fand allerdings keine weite Verbreitung, da nur geringe und variable Ausbeuten erzielt werden konnten. Außerdem erfolgte der Nachweis der entstandenen Aminosäuren meist nur semiquantitativ mittels Papier- und Dünnschichtchromatographie. Auch die elektrolytische Hydrierung, wie sie von SMITH (11) vorgeschlagen wurde, brachte keine befriedigenden Resultate.

Wir sind bei unseren Untersuchungen von der Methode von Towers und Mitarbeitern (9) ausgegangen und sind dabei wie folgt verfahren:

1. Hydrazonbildung - Durchführung vergleichender Untersuchungen bei verschiedenen $\mathrm{pH}$-Werten, Temperaturen und 2,4-Dinitrophenylhydrazon-Konzentrationen sowie mit verschiedenen Phenylhydrazinen

2. Hydrierung - Test der Hydrierungsbedingungen bei verschiedenen $\mathrm{pH}$-Werten, Temperaturen und Lösungsmitteln

3. Analyse - Bestimmung der entstehenden Aminosäuren mittels Kolonnen-Chromatographie nach STEIN und MOORE. 


\section{Material und Methoden}

Gerätc

Aminosäuren-Analysator Bio-Cal Modell „Biochrom BC-200“; Hydrierungsapparatur (siehe Abb. 1).

\section{Chemikalien}

Referenz-Oxosäuren: Sigma Chemical Company, St. Louis, Mo. (USA); 2,4-Dinitrophenylhydrazin: Fluka AG, Buchs (Schweiz). Platinoxid: Fluka AG, Buchs (Schweiz).

Wasserstoff reinst: SWWL, Luzern (Schweiz).

Lösungsmittel

Fluka AG, Buchs (Schweiz) und Merck AG, Darmstadt (BRD). Sämtliche Lösungsmittel waren von p. a. Qualität oder wurden vor Gebrauch destilliert.

\section{Reagenzien}

Testlösung: Je $100 \mathrm{mg}$ folgender Säuren als Natriumsalz: Brenztraubensäure, Phenyl-brenztraubensäure, $p$-Hydroxy-phenylbrenztraubensäure, 2-Oxo-glutarsäure, 2-Oxo-bernsteinsäure, 2-Oxo-ncapronsäure, 2-Oxoisocapronsäure, 2-Oxo-3-methylvaleriansäure, 2-Oxo-isovaleriansäure, 2-Oxo-buttersäure und Glyoxylsäure in dest. Wasser ad $100 \mathrm{ml}$ (bei $4^{\circ}$ aufbewahren).

Gesättigte Lösung von 2,4-Dinitrophenylhydrazin in $5 \mathrm{~N} \mathrm{HCl}$; $0,2 \mathrm{~N}$ Citratpuffer ( $\mathrm{pH} 2,2$ ) zum Beschicken des AminosäurenAnalysators.

Sammeln des 24-Stdn.-Urins.

Bei $4^{\circ}$ unter Hinzufügen von etwas Chloroform (sofort aufarbeiten).

\section{Arbeitsvorschrift}

Pbenglly'drazon-Bildung: 10 bis $50 \mathrm{ml}$ Urin werden mit 2 bis $10 \mathrm{ml}$ einer gesättigten 2,4-Dinitrophenylhydrazon-Lösung in $5 \mathrm{~N} \mathrm{HCl}$ versetzt; das $\mathrm{pH}$ soll zwischen 2,5 und 3 liegen. Anschließend wird 10 bis $15 \mathrm{Stdn}$. bei $4^{\circ}$ im Dunkeln inkubiert. Die gelb gefärbten Hydrazone fallen in wäßr. Medium als schwer lösliche Verbindungen aus. $\mathrm{Da}$ ein beträchtlicher Teil der Substanzen in Lösung : bleibt, empfiehlt es sich, nicht abzuzentrifugieten. Die Lösung wird deshalb mit je $20 \mathrm{ml}$ eines EssigsäureäthylesterÄther Gemisches $(1: 1 \mathrm{v} / \mathrm{v})$ mehrmals extrahiert, bis das Extraktionsmittel farblos bleibt. Sollten sich bei der Extraktion Suspensionen bilden, so erreicht man eine bessere Trennüng der Phasen durch Zugabe von etwas Äther oder Essigsäureäthylester. Die vereinigten Extrakte werden im Rotationsverdampfer eingedampft, wobei das Wasserbad eine Temperatur von $30^{\circ}$ nicht übersteigen soll. Dem fast zur Trockene eingedampften Präparat fügt man etwas Äthanol zu, um mitgeführtes Wasser azeotrop zu entfernen.

Präparative Darstellung der 2,4-Dinitrophenylbydrazone von $p$-Hydroxjphenylbrenztraubensäure, Brenztraubensäure und 2-Oxoglutarsäure: Die Phenylhydrazone wurden aus den Testsubstanzen nach obiger Vorschrift synthetisiert und mittels Elementaranalyse und Massenspektrometrie charakterisiert.

Hydrierıng: Der Rückstand wird in $5 \mathrm{ml}$ Äthanol aufgenommen und die Lösung mit einigen $\mu \mathrm{l} \mathrm{NaOH}$ auf einen $\mathrm{pH}$-Wert von 6,5 bis 7,5 gebracht. 1 bis $4 \mathrm{ml}$ der auf 5 bis $10^{\circ}$ temperierten Lösung werden mit $5 \mathrm{mg} \mathrm{PtO}_{2}$ versetzt, mit Äthanol auf $6 \mathrm{ml}$ aufgefüllt und in einem Schliffröhrchen von $1 \mathrm{~cm}$ Durchmesser und $10 \mathrm{~cm}$ Länge so lange hydriert, bis die gelbe Farbe über dunkelgelb nach hellgelb und schließlich nach farblos umschlägt. Die Entfärbung erfolgt je nach Konzentration der Phenylhydrazone in 20 bis $30 \mathrm{Min}$. Zur Hydrierung wurde die in Abbildung 1 dargestellte Apparatur verwendet.

Der Wasserstoff wird in einem Schliffrohr von $1 \mathrm{~cm}$ Durchmesser und $10 \mathrm{~cm}$ Länge durch die zu hydrierende Lösung geleitet (Verdampfungskälte bewirkt Abkühlung auf 5 bis $10^{\circ}$ ). Nach beendigter Hydrierung wird der Katalysator abzentrifugiert und die Lösung in Rotations-Verdampfer eingedampft.

Quantitative Aminosäuren-Analyse: Der Rückstand wird in einem Puffer von $\mathrm{pH} 2,2$ gelöst und auf die sauer-neutrale Säule des Aminosäuren-Analysators aufgebracht. Die Säule wird vorher wie

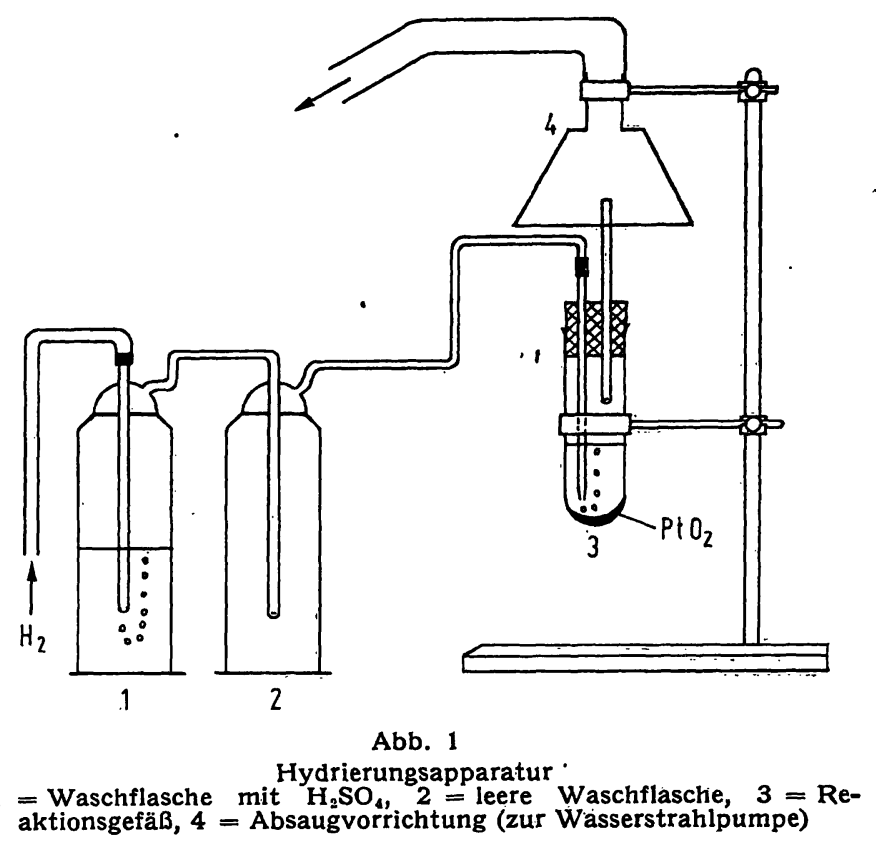

folgt präpariert: Am oberen Ende des Ionenaustauscherharzes wird zunächst eine Teflonfritte angebracht, die mit Dowex $50 \times 8$ 100 bis 200 mesh Kationenaustauscher $2 \mathrm{~cm}$ hoch überschichtet wird. Vor dem Auftragen der zu untersuchenden AminosäureLösung wird die gesamte Kolonne mit $0,2 \mathrm{~N} \mathrm{NaOH}$ gewaschen und mit Puffer $\mathrm{pH}$ 3,25 äquilibriert. Nach Beendigung der Analyse, vor der Regeneration mit $1 \mathrm{~N} \mathrm{NaOH}$, saugt man die aufgebrachte Harzschicht über der Teflonfritte ab, um eine Kontamination des teuren Aminosäure-Harzes mit Spaltprodukten (Farbstoffen) zu vermeiden.

\section{Resultate}

Beim Vergleich verschiedener Derivate (Phenylhydrazone, $p$-Nitrophenylhydrazone und 2,4-Dinitrophenylhydrazone) ergab sich für die 2,4-Dinitrophenylhydrazone die beste Ausbeute. Die Phenylhydrazonbildung erfolgt nach folgender Formel:

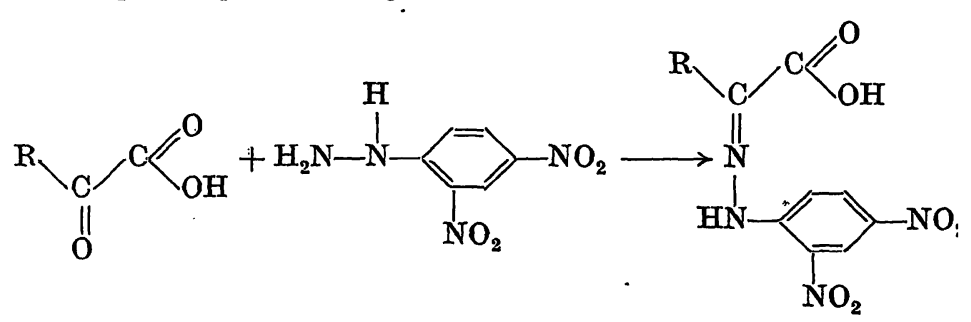

Als Konkurrenzreaktion kann in saurer Lösung nach folgendem Schema Decarbonylierung eintreten:<smiles>[R]C(=O)C(=O)OCC(=O)O</smiles>

Die genauen Reaktionsbedingungen wie pH, Temperatur und Konzentration des Reagenz sind daher für die Ausbeute von großer Bedeutung. Als günstigste Bedingung erwies sich die Verwendung einer gesättigten 2,4-Dinitrophenylhydrazin-Lösung in $5 \mathrm{~N} . \mathrm{HCl}$, was im Urin $\mathrm{pH} 2,5$ bis 3 ergibt. Die Abhängigkeit von Zeit und Temperatur ist in Abbildung 2 dargestellt.

Die Hydrierung der 2,4-Dinitrophenylhydrazone erfolgt nach folgendem Reaktionsschema: 
Philips bietet ein gut sortiertes Programm an Elektroden mit hoher Selektivität, hoher Empfindlichkeit und hoher Stabilität

mit Festkörpermembran Chlorid, Bromid, Jodid, Cyanid, Sulfid,

Fluorid, Silber, Cadmium

mit Flüssigmembran

Heute schon ist es in vielen Labors wünschenswert, lonenaktivitäten messen zu können. Dafür stehen universelle Geräte zur Verfügung, die den höheren Anforderungen gerecht werden - für alle lieferbaren ionenselektiven Elektroden, z. B. zur Messung von $\mathrm{pCl}-, \mathrm{pCN}$-, pCa- und $\mathrm{pNH}_{4}$-Werten. Selbstverständlich lassen sich damit auch pH-Werte und Redoxpotentiale messen. AuBerdem - und das ist interessant - sind diese Geräte zusätzlich für amperometrische Titrationen eingerichtet. Der finanzielle Aufwand ist gering, der Zuwachs an Anwendungsmöglichkeiten und Flexibilität dagegen erfreulich hoch.

Das Ionenaktivitäts-MeBgerät PW 9413 für ionenspezifische Messungen in Industrie und Forschung, z. B. bei der Wasser- und Abwasserüberwachung und -untersuchung - dabei sind kontinuierliche Kontrollmessungen möglich - und

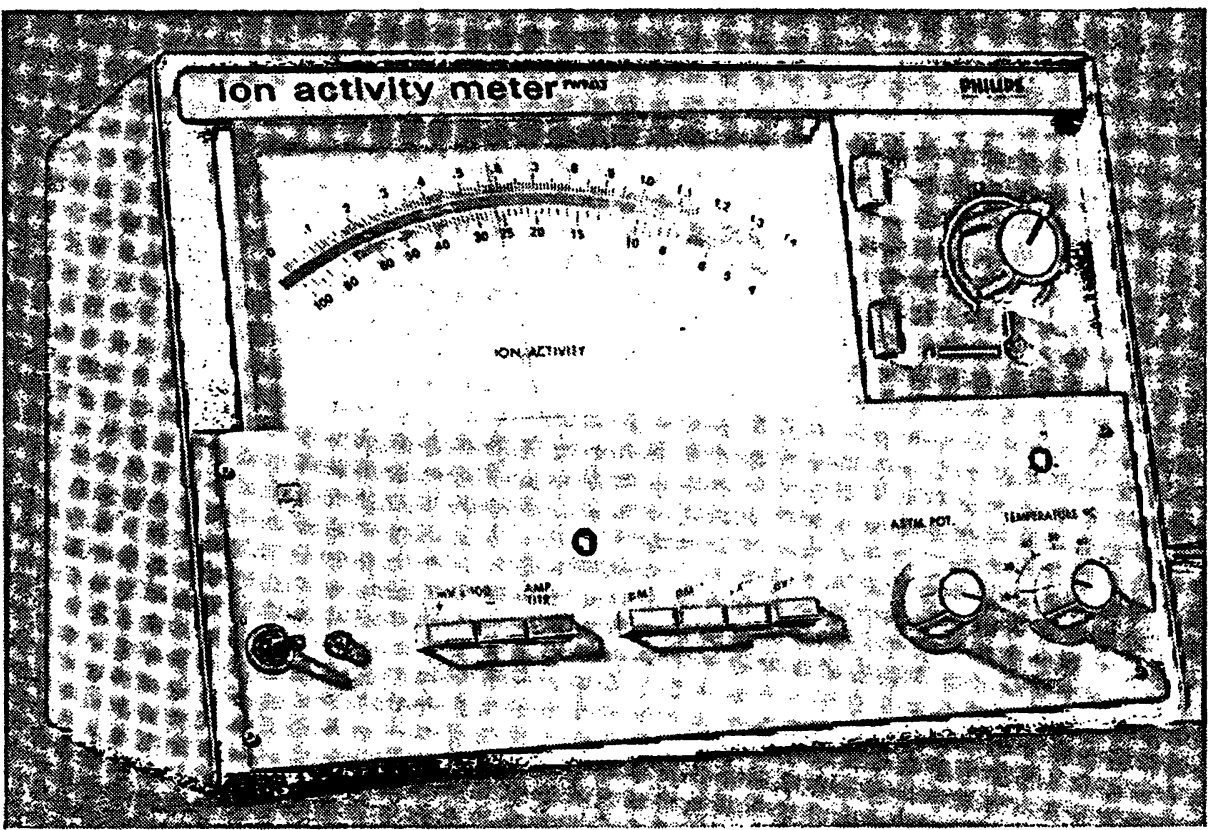

in der klinischen Chemie, z. B. bei der Blut-, Serum-, Speichel- und Harnuntersuchung:

Dạs Gerät hat eine hohe Empfindlichkeit Dabei ist die Ablesegenauigkeit auf der $190 \mathrm{~mm}$ langen Skala bereits ungewöhnlich gut; sie läßt sich durch Bereichsspreizung noch vergrößern.

... und eine hohe Stabilität

Dafür sorgt der Brückeneingang mit Varaktordioden.

\section{Die Bedienung ist einfach}

Die Bedienungselemente sind übersichtlich angeordnet. Das macht die Arbeit leicht und bequem.

\section{Ein Knopidruck genügt,}

um ein- oder zweiwertige Anionen und Kationen vorzuwählen. Die Ionenaktivität bzw: -konzentration wird dann direkt auf dem Meßgerät abgelesen.
Sogar bei amperometrischen Titrationen genügt ein Knopfdruck. Das Meßgerät zeigt dann den Strom in $\mu \mathrm{A}$ an - direkt geeignet für Karl Fischer Titrationen. Die Gleichspannung ist einstellbar zwischen 0 und $100 \mathrm{mV}$ Asymmetriepotential-Einstellung:

$-300 \ldots+300 \mathrm{mV}$

Steilheltskorrektur: $54,0 \ldots 59,2 \mathrm{mV}$ für einwertige und $27,0 \ldots 29,6 \mathrm{mV}$ für zweiwertige lonen

Temperaturkompensation von $\mathrm{Hand}$ oder automatisch mit Pt $100 \mathrm{Ohm}$ Widerstandsthermometer

Reproduzierbarkeit $\pm 0,2 \%$ vom Skalenendwert
Kalium, Ammonium, Calcium mit Glasmembran

Kalium, Natrium - auch EinstabMeßketten

Referenzelektroden

mit Elektrolytbrücke, speziell für Messung mit ionenselektiven Elektroden

Weitere Elektroden, z. B: für Schwermetalle, sind in Vorbereitung

zur weiteren Information schickt Ihnen Philips gern ausführliches Informationsmaterial. Bitte fordern Sie es an.

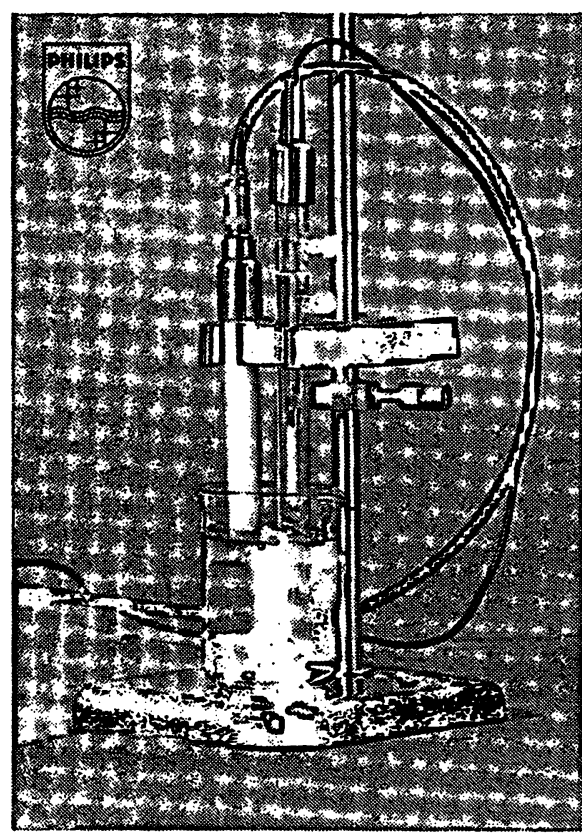

Philips Elektronik Industrie GmbH 2000 Hamburg 63, Röntgenstraße 22 Telefon (0411) 580131

Telefon-Nummern der Büros In: Berlin (0311) 2459 o 8 , Bielefeleld 34 60 51/55, Frankturt (0611) 790131 , Hamburg (0411) 501031 Hannover (0511) 166 O1. Klel $(0431)$ i3 $2386 . \mathrm{KoIn}(0221) 514260$ Mannhelm (0621) 42016 18. Münnchen (0811) 76791 , Nürnberg

in Österreich: Österreichische Philips Industrie GmbH, Wien, Triester Str. 64 in der Schweiz: Philips AG, Zürich,

Postfach, Tel.: (051) 442211

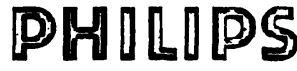

Wir interessieren uns für das Meßgerät PW 9413 und für ionenselektive Elektroden und bitten um

$\square$ Zusendung ausführlicher Unterlagen

$\square$ ein Angebot:

Gewünschtes bitte ankreuzen oder ergänzen 





<smiles>[R]C(=NNc1ccc([N+](=O)[O-])cc1[N+](=O)[O-])C(=O)O</smiles><smiles>[R]C(NNc1ccc([N+](=O)[O-])cc1[N+](=O)[O-])C(=O)O</smiles><smiles>[R]C(N=[NH2+])C(=O)O</smiles>

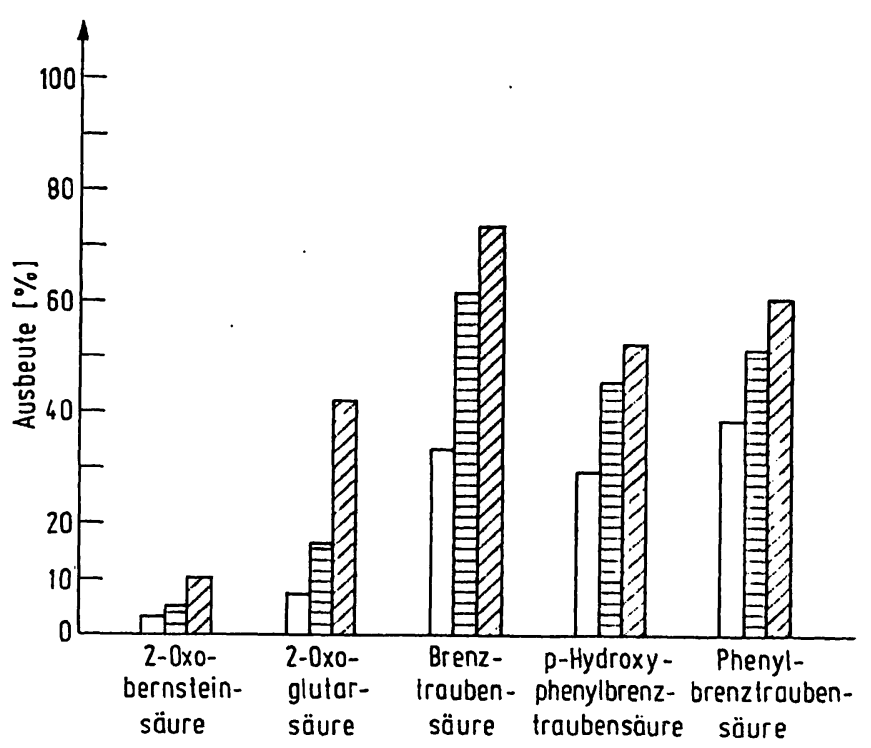

Abb. 2

Diagramm der Gesamtausbeuten bei verschiedenen Reaktionsbedingungen für die Hydrazonbildung bei pH 2,5 (weiße Säulen: 30 Min. bei $40^{\circ}$, horizontal schraffierte Säulen: $12 \mathrm{Stdn}$. bei $24^{\circ}$, schräg schraffiérte Sâulen: 12 Stan. bei $4^{\circ}$ ); Hydrierung bei pH' 7 und $15^{\circ}$

Wir haben die Hydrierung in verschiedenen Lösungsmitteln, bei verschiedenem $\mathrm{pH}$ und unter verschiedenen Hydrierungsbedingungen überprüft. Die katalytische Hydrierung in Äthanol eignet sich besser als diejenige in Wasser (12). Hydrierungsversuche bei unterschiedlichem $\mathrm{pH}$ (alkalisch, sauer und neutral) ergaben die besten Ausbeuten bei einem pH-Wert von 6,5 bis 7,5. Die von Towers, THOMPSON und STEWARD verwendete Hydrierungsmethode mittels einer Apparatur nach PARR benötigt bis zur Beendigung der Reaktion $\mathrm{Hy}$ drierungszeiten bis zu $12 \mathrm{Stdn}$. bei Raumtemperatur. Hierbei können in erheblichem Maße Nebenreaktionen ablaufen. Beim Durchleiten von Wasserstoff durch die Lösung in der Kälte ist die Hydrierung bereits nach 20 bis $30 \mathrm{Min}$. beendet, und es werden daher weniger Zersetzungsprodukte gebildet. Ein Vergleich der Hydrierung bei verschiedenen Temperaturen ergab die besten Bedingungen bei 5 bis $10^{\circ}$. In Abbildung 3 ist die Abhängigkeit der Hydrierung von $\mathrm{pH}$ und Temperatur dargestellt.

Die elektrolytische Hydrierung nach SMITH (11) wurde von uns ebenfalls getestet. Wir benutzten dazu eine Apparatur mit einer semipermeablen Membran und zwei Platinelektroden (Durchfluß).

Es ergaben sich hierbei apparative Schwierigkeiten (Wärmeentwicklung) und Probleme der $\mathrm{pH}$-Einstellung. Für die elektrolytische Reduktion benötigen wir
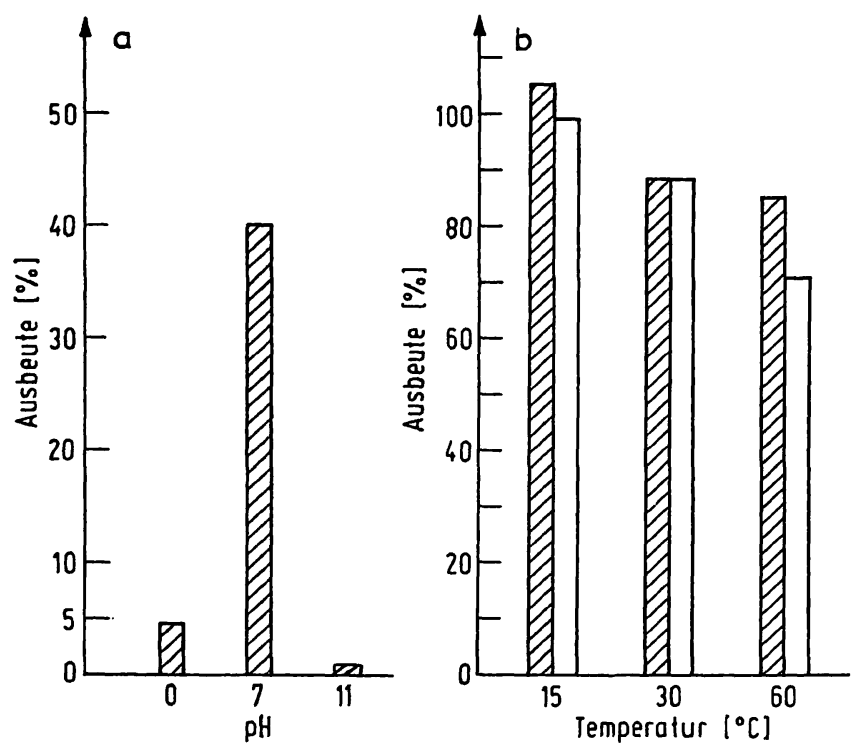

Abb. 3

Diagramm der Ausbeuten der katalytischen Reduktion bei verschie-

denen Reaktionsbedingungen
a) $p$-Hydroxyphenylbrenztraubensäure bei $20^{\circ}$ b) Brenztraubensäure (schraffiert) und 2-Oxoglutarsäure (weiß) bei

Protonen, andererseits begünstigt ein saures $\mathrm{pH}$ die Decarbonylierung.

In Tabelle 1 sind Ausbeute und Variationskoeffizient von 10 Parallelbestimmungen elf verschiedener Oxosäuren dargestellt. Die Oxosäuren wurden jeweils vor Beginn der Analyse dem Urin beigemischt.

Tab. 1

Wiederauffindungs- und Reproduzierbarkeits-Versuche von 11 dem Urin zugemischten Oxosäuren (jeweils 12 Parallelbestimmungen)

\begin{tabular}{lccc}
\hline \multicolumn{1}{c}{$\begin{array}{c}\text { Testsubstanz } \\
\text { (Oxosäure) }\end{array}$} & $\begin{array}{c}\text { Bestimmung } \\
\text { als Aminosäure }\end{array}$ & $\begin{array}{c}\text { Ausbeute } \\
(\%)\end{array}$ & $\begin{array}{c}\text { Variations- } \\
\text { koeffizient } \\
(\%)\end{array}$ \\
\hline $\begin{array}{l}\text { Brenztraubensäure } \\
\text { Phenylbenz- }\end{array}$ & Alanin & $79,1 \pm 9,5$ & 12,0 \\
traubensäure & Phenylalanin & $50,0 \pm 8,0$ & 16,1 \\
$\begin{array}{l}\text { p-Hydroxy- } \\
\text { phenylbrenz- }\end{array}$ & Tyrosin & $43,0 \pm 7,2$ & 16,6 \\
$\begin{array}{l}\text { traubensäure } \\
\text { 2-Oxo-glutarsäure }\end{array}$ & Glutaminsäure & $48,8 \pm 8,5$ & 17,3 \\
$\begin{array}{l}\text { Glyoxylsäure } \\
\text { 2-Oxo-buttersäure }\end{array}$ & $\begin{array}{c}\text { Glycin } \\
\text { 2-Oxo-isovalerian- }\end{array}$ & $30,8 \pm 5,9$ & 19,0 \\
$\begin{array}{l}\text { säure } \\
\text { 2-Oxo-3-methyl- }\end{array}$ & Valin & $58,2 \pm 4,9$ & 8,3 \\
valeriansäure & Isoleucin + allo-Isoleucin & $56,2 \pm 7,1$ & 12,5 \\
$\begin{array}{l}\text { 2-Oxo-isocapron- } \\
\text { säure }\end{array}$ & Leucin & $68,1 \pm 9,8$ & 14,4 \\
$\begin{array}{l}\text { 2-Oxo-capronsäure } \\
\text { 2-Oxo-bernstein- } \\
\text { säure') }\end{array}$ & Norleucin & $56,1 \pm 8,6$ & 15,2 \\
\hline
\end{tabular}

1) decarboxyliert sehr leicht 

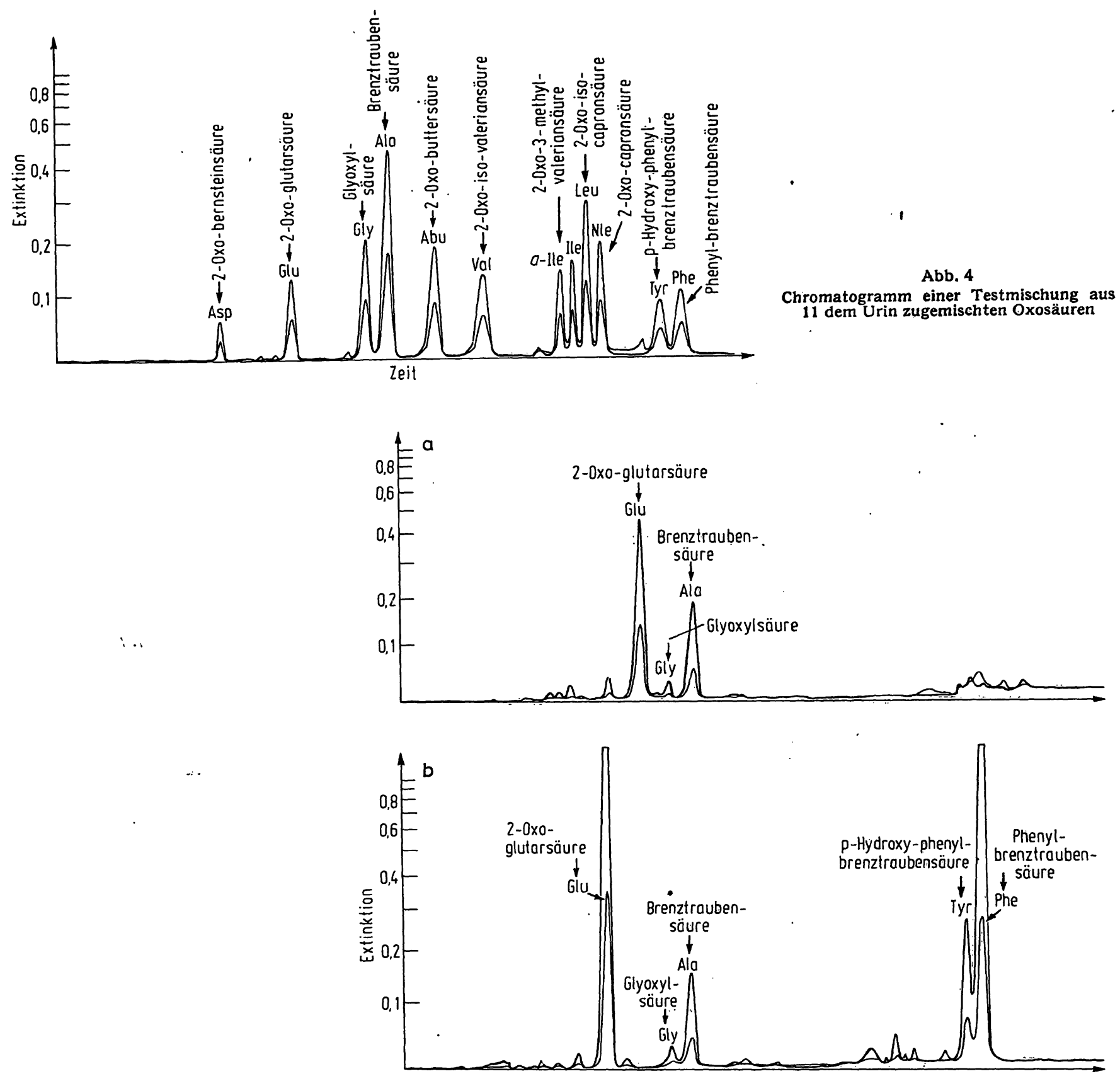

Abb. 5

Chromatogramm eines gesunden Probanden (a) eines Patienten mit Phenylketonurie

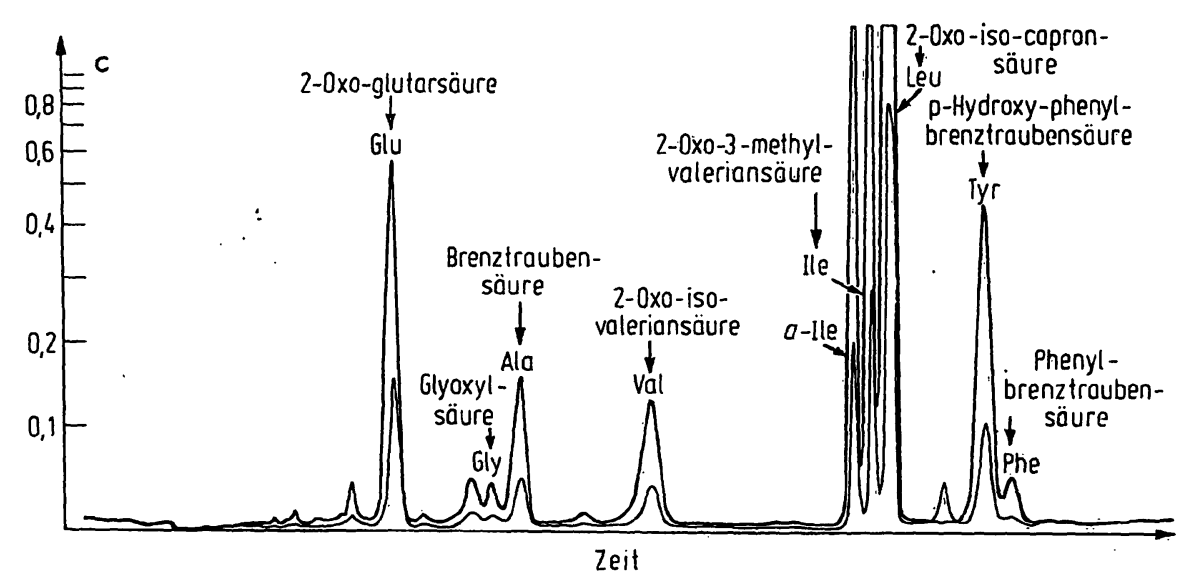

Abbildung 5 zeigt das Chromatogramm eines Gesunden, eines Patienten mit Phenylketonurie und eines Patienten mit Leucinose.
In Abbildung 4 ist ein entsprechendes AminosäurenChromatogramm dargestellt. 


\section{Diskussion}

Bei der Bildung der Phenylhydrazone sind die 2,4Dinitrophenylhydrazone die geeignetsten Derivate. Die Zugabe einer gesättigten Lösung von 2,4-Dinitrophenylhydrazin in $5 \mathrm{~N} \mathrm{HCl}$ und eine 10 - bis 15 stdg. Inkubation in der Kälte erwiesen sich als die besten Reaktionsbedingungen. Die Hydrierung in Äthanol ist derjenigen in Wasser überlegen. Beim Durchleiten von Wasserstoff bei 5 bis $10^{\circ}$ kann die Reaktionszeit auf 20 bis 30 Min. beschränkt und damit die Entstehung von Nebenprodukten weitgehend vermieden werden. Die Úberschichtung des teuren sphärischen Aminosäuren-Harzes mit gewöhnlichem Kationenaustauscher verhindert die Kontamination der Säule, und es wird dadurch die quan̨titative Aminosäuren-Analyse mit einem der üblichen Analysatoren möglich. $\mathrm{Da}$ die Ausbeuten nicht noch höher sind, ist im wesentlichen auf die Phenylhydrazonbildung zurückzuführen. Die katalytische Hydrierung verläuft nahezu quantitativ. Mit Hilfe der so abgeänderten Methode können praktisch alle Oxosäuren mit relativ guter Ausbeute und hoher Empfindlichkeit und Spezifität bestimmt werden. Allerdings weist die Oxalessigsäure aufgrund ihrer leichten Decarboxylierbarkeit eine sehr geringe Ausbeute auf. Der Arbeitsaufwand der Methode ist relativ klein. Limitierender Faktor ist lediglich die Aminosäuren-Analyse, wobei jedoch bei Beschränkung auf einige bestimmte Oxosäuren mit Hilfe von Aminosäuren-Kurzprogrammen (13-15) die Analysendauer wesentlich reduziert werden kann.

\section{Literatur}

1. Cavallini, D., N. Frontali und G. Toschi, Nature London 163, 568 (1949). - 2. Coward, R. F. und P. Smith, J. Chromatog. 33, 508 (1968). - 3. Berlet, H. H., Analytic. Biochem. 22, 525 (1968). - 4. Lutz, P. und G. M. v. ReuterN, diese Z. 7, 586 (1969). - 5. Horning, E. C. und M. G. Horning, J. Chromatog. Sci. 9, 129 (1971). - 6. Hoffman, N. E., K. M. Gooding, K. M. Sheahan und C. A. Tylenda, Research Communications in: Chem. Pathol. Pharmacol. 2, 87 (1971). - 7. Kesner, L. und E. Muntwyler, Analytic. Chem. 38, 1164 (1966). - 8. RonkaInen, P., J. Chromatog. 28, 263 (1967). - 9. Towers, G. H. N., F. C. Steward, und J. F. Thompson, J. Amer. chem. Soc. 76 ,
2392 (1954). - 10. Meister, A. und P. A. Anendschein, Analytic. Chem. 28, 171 (1956). - 11. SMrth, I. in: Chromatographic and Electrophoretic Techniques, Vol. 1, William Heinemann Medical Books Ltd., London (1969). - 12. Grob, C. A., in: Synthetische Methoden der organischen Chemie, Verband Basler Chemiestudierender, 1959. - 13. Benson, J. V., J. Cormik und J. A. Patterson, Analytic. Biochem. 18, 481 (1967). - 14. Shin, V. E., M. L. Erron und G. L. Mechanic, Analytic. Biochem. 20, 299 (1967). - 15. Hamilton, P. B., in: Handbook of Biochemistry, Selected Data for Molecular Biology, S. B-43-B-55 (Editor: H. A. Sober), The Chemical Rubber Co., Cleveland, Ohio (1968).
Priv.-Doz. Dr. H.-Ch. Curtius Steinwiesstr. 75 $\mathrm{CH}-8032$ Zürich 